\title{
PARASITISMO DE TRICHOGRAMMA PRETIOSUM RILEY, 1879 (HYMENOPTERA: TRICHOGRAMMATIDAE) EM DIFERENTES HOSPEDEIROS E CORES DE CARTELAS
}

\author{
G.O. Magalhães, R.M. Goulart, A.M. Vacari, S.A. De Bortoli
}

Universidade Estadual Paulista, Faculdade de Ciências Agrárias e Veterinárias, Departamento de Fitossanidade, Laboratório de Biologia e Criação de Insetos, Via de Acesso Prof. Paulo Donato Castellane, s/no, CEP 14884-900, Jaboticabal, SP, Brasil. E-mail: godemagalhaes@uol.com.br

\section{RESUMO}

Espécies do gênero Trichogramma são dos inimigos naturais os mais estudados e utilizados atualmente no mundo. A qualidade e o desempenho desses agentes de controle produzidos massalmente podem ser influenciados por alguns fatores, tais como a escolha do hospedeiro adequado para multiplicação do parasitoide e a utilização de diferentes cores de cartelas, haja vista que estes parasitoides podem utilizar estímulos físicos como cor para facilitar a localização do hospedeiro. $\mathrm{O}$ objetivo desse trabalho foi avaliar à influência de cores de cartelas no parasitismo de Trichogramma pretiosum que foi submetido a ovos do hospedeiro alternativo Anagasta kuehniella e duas populações de Plutella xylostella, sendo uma delas criada no substrato brócolis e a outra em couve. Ovos dos hospedeiros foram aderidos às cartelas de papel cartão, sendo que as cores selecionadas foram: azul, amarela, verde, rosa, vermelha e preta. Para cada tratamento foram observadas 20 repetições, cada uma composta por 30 ovos dos hospedeiros. Posteriormente, as cartelas foram expostas às fêmeas do parasitoide individualizadas em tubos tipo Eppendorf ${ }^{\circledR}$ de 2,0 $\mathrm{mL}$ que continha uma gotícula de mel para alimentação delas por 24 horas, em teste de confinamento. Foram avaliados o número de ovos parasitados e a porcentagem de emergência. A linhagem Tp-8 de T. pretiosum apresentou maior parasitismo em ovos de A. kuehniella. Os substratos de alimentação de $P$. xylostella (couve e brócolis) não influenciam o parasitismo de T. pretiosum. As cores de cartelas indicadas para a condução da criação massal de T. pretiosum são azul, verde e branco.

PALAVRAS-CHAVE: Parasitoide de ovos, criação massal, controle biológico.

\begin{abstract}
INFLUENCE OF COLOR CARDS, HOSTS AND FIRST TROPHIC LEVEL ON TRICHOGRAMMA PRETIOSUM RILEY, 1879 (HYMENOPTERA: TRICHOGRAMMATIDAE) PARASITISM. Species of the Trichogramma genus are the most studied and used natural enemies worldwide. The quality and performance of the mass-produced control agents can be influenced by some factors such as the choice of the appropriate host for multiplication of the parasitoid and the use of different colors of cards, given that these parasitoids may use physical stimuli such as color to help in locating the host. The aim of the present study was to evaluate the influence of color cards on the parasitism of Trichogramma pretiosum in eggs of Anagasta kuehniella and in eggs of two populations of Plutella xylostella, one reared on broccoli, the other on collard greens. Hosts' eggs were stuck to the different color cards. The selected colors were blue, yellow, green, pink, red and black. For every treatment, 20 replications were observed, and each was composed by 30 eggs of those hosts. Later the cards were exposed to parasitoid females individually in Eppendorf ${ }^{\circledR}$ tubes of $2.0 \mathrm{~mL}$ containing one honey droplet for feeding for 24 hours. The number of parasitized eggs and the emergence percentage were evaluated. The strain $\mathrm{Tp}-8$ of T. pretiosum presented better greater parasitism in A. kuehniella eggs. The feeding substratum of $P$. xylostella (collard greens and broccoli) did not influence the parasitism of $T$. pretiosum. Thus for mass rearing of $T$. pretiosum, blue, green and white color cards are most suitable.
\end{abstract}

KEY WORDS: Egg parasitoids, mass rearing, biological control.

\section{INTRODUÇÃO}

Dentre os parasitoides de ovos, os do gênero Trichogramma têm sido os mais estudados eutilizados em programas de controle biológico, devido a sua eficiência, ampla distribuição geográfica efacilidade de criação em laboratório. Esses parasitoides, por atacarem ovos, impedem que seus hospedeiros, 
principalmente lepidópteros-praga, atinjam a fase de larva, na qual podem causar danos econômicos às culturas (HAJi et al., 1995).

Em todo o mundo são conhecidas, aproximadamente, 180 espécies do gênero Trichogramma (PINTO, 1999). No Brasil, estão registradas 25 espécies distribuídas em quase todas as regiões (QuERINO; ZucchI, 2003). Trichogramma pretiosum Riley, 1879 (Hymenoptera: Trichogrammatidae) é a mais distribuída ejá foi relatada em 18 diferentes hospedeiros em 13 culturas (Zucchi; MonteIro, 1997; Goulart et al., 2008).

A partir da década de trinta ocorreu um avanço significativo na utilização de Trichogramma, quando FLANDERS (1927) divulgou um método de criação massal utilizando como hospedeiro alternativo ovos de Sitotroga cerealella (Olivier, 1819) (Lepidoptera: Gelechiidae). Essa estratégia de criação massal tornou-se rotineira e a multiplicação de espécies de Trichogramma em larga escala, também em ovos de outros hospedeiros, como Anagasta kuehniella (Zeller, 1879) (Lepidoptera: Pyralidae), expandiu-se (PARRA, 1997). Atualmente, esses parasitoides podem ser produzidos em hospedeiros naturais ou alternativos ou em dietas artificiais à base de hemolinfa de insetos (PARRA et al., 2002).

No entanto, o sucesso ou fracasso da utilização de parasitoides do gênero Trichogramma no controle de lepidópteros-praga relaciona-se com o conhecimento das características biológicas deste parasitoide como: capacidade e viabilidade do parasitismo, duração do ciclo de desenvolvimento, razão sexual e longevidade, sendo essas características altamente influenciadas pelas condições bióticas e abióticas (FUENTES, 1994).

A espécie T. pretiosum tem sido mencionada em diversos países como importante agente de controle de Plutella xylostella (Linnaeus, 1758) (Lepidoptera: Plutellidae) (Goulart et al., 2012). No Brasil, são poucas as pesquisas que relacionam esse parasitoide à traça-das-crucíferas.

Os parasitoides podem identificar estímulos físicos como cor, tamanho, forma, som e vibração como pistas para localização do hospedeiro (VINSON, 1985). Entre estes estímulos, a cor destaca-se tanto como pista direta, quanto através de aprendizagem associativa em relação à localização do habitat e do hospedeiro (SEgura et al., 2007). No contexto de pistas relacionadas ao forrageamento de parasitoides, a cor é um fator que tem sido estudado, entretanto, foram realizados estudos com parasitoides da família Scelionidae (AQuINo, 2011), existindo menor volume de informação no caso de Trichogrammatideos.

Dessa forma, este trabalho teve como objetivo avaliar a influência de cores de cartelas para criação massal de T. pretiosum utilizando ovos do hospedeiro alternativo A. kuehniella, e do hospedeiro natural
P. xylostella, sendo este criado em dois substratos, couve e brócolis.

\section{MATERIAL E MÉTODOS}

As criações dos insetos foram mantidas no Laboratório de Biologia e Criação de Insetos (LBCI) do Departamento de Fitossanidade da FCAV/Unesp, campus de Jaboticabal. P. xylostella foi conduzida segundo THULER (2009), e T. pretiosum foi conduzida de acordo com PARRA (1997) modificada por GOULART (2009).

O experimento foi mantido a $25 \pm 1^{\circ} \mathrm{C}$, fotofase de $12 \mathrm{~h}$ e UR de $70 \pm 10 \%$, em esquema fatorial utilizando-se como hospedeiros ovos de A. kuehniella, ovos de $P$. xylostella oriundos de criações mantidas com substrato couve e brócolis; para a fixação dos ovos de cada hospedeiro foram utilizadas cartelas de cores azul, amarela, verde, rosa, vermelha e preta. Para cada tratamento foram observadas 20 repetições e para cada uma delas foram utilizados aproximadamente 100 ovos de A. kuehniella, delimitados em área de $0,2 \mathrm{~cm}^{2}$, aderidos nas cartelas $(0,4 \times 2,0 \mathrm{~cm})$ utilizando-se goma arábica a $10 \%$. Os ovos de P. xylostella (30 ovos por repetição) foram retirados de criações mantidas em folhas de couve e de brócolis e, posteriormente, foram aderidos nas cartelas com auxílio de pincel de ponta fina. O número de hospedeiros oferecidos foi diferente porque essa metodologia de delimitar uma área de $0,2 \mathrm{~cm}^{2}$ onde para colagem de ovos de A. kuehniella é frequentemente utilizada com sucesso em experimentos com Trichogramma (VolPE et al., 2006; GOULART et al., 2012); foram utilizados 30 ovos de P. xylostella porque foi observado em outros trabalhos que o número de ovos parasitados desse hospedeiro nunca ultrapassa 30 por fêmea, independe da espécie de Trichogramma utilizada e das condições bióticas e abióticas (Pratissoli et al., 2004; Pereira et al., 2004; POLANCZYK et al., 2007; THULER et al., 2008). As cartelas foram expostas a uma fêmea de T. pretiosum em tubos tipo Eppendorf $^{\circledast}$ de 2,0 mL que continham uma gotícula de mel para alimentação do parasitoide. Após 24 h, a fêmea foi retirada dos tubos e as cartelas foram mantidas em condições controladas a $25 \pm 1^{\circ} \mathrm{C}$, fotofase de $12 \mathrm{~h}$ e UR de $70 \pm 10 \%$, para posterior avaliação do número de ovos parasitados e da porcentagem de emergência.

Os dados foram submetidos à análise exploratória para a determinação da homogeneidade, normalidade e padrão de distribuição de resíduos. Os procedimentos estatísticos, análise de variância e teste de média (Tukey $\mathrm{P}<0,007$ ) foram realizados utilizando o programa SAS Version 9.00 (SAS INSTITUTE, 2002). 


\section{RESULTADOS E DISCUSSÃO}

Não houve diferença significativa no número de ovos parasitados entre as diferentes cores de cartelas utilizando ovos de P. xylostella independente da fonte de alimentação dos insetos (brócolis $\mathrm{F}_{6,85}=2,28$; $\mathrm{P}=0,0445$ e couve $\mathrm{F}_{6,90}=1,05 ; \mathrm{P}=0,3972$ ) (Tabela 2). Enquanto que com ovos de A. kuehniella $\left(\mathrm{F}_{6,101}=\right.$ 4,$37 ; \mathrm{P}=0,0006$ ) houve diferença entre as cores de cartelas e dentre as utilizadas a de cor preta obteve menor parasitismo (Tabela 2). Para todas as cores de cartelas, exceto a cor preta, o número de ovos parasitados foi maior com A. kuehniella $(15,5)$, em relação aos tratamentos com ovos de $P$. xylostella $(5,3)\left(F_{1,6}=118,41 ; P<0,0001\right)$ (Tabelas 1 e 2$)$.

As cores apresentam três principais características: intensidade (energia refletida), matiz (comprimento de onda dominante) e saturação (pureza do espectro da luz refletida) (BERNAYS; CHAPMAN, 1994). Insetos fitófagos utilizam essas três características, sozinhas ou juntas para orientação, como, por exemplo, a visão de cores em Hymenoptera que é influenciada pela intensidade (Hollingsworth et al., 1970) e saturação (LuNAU, 1990). Em armadilhas adesivas de diferentes cores espécies de Trichogramma podem utilizar interpretações visuais durante $o$ processo de localização do habitat do hospedeiro (RomeIs et al., 1998).

Outro estudo que observou o parasitismo de $T$. pretiosum em diferentes hospedeiros verificou média de10,8 e 15,6 ovos parasitadosem cartelas de coloração azul para P. xylostella e A. kuehniella, respectivamente (VolPe et al., 2006). Thuler et al. (2007) também observaram que T. pretiosum apresentou melhor adaptação ao hospedeiro alternativo A. kuehniella.
Um fator que poderia explicar o menor número de ovos parasitados nos tratamentos com $P$. xylostella seria quea taxade parasitismode T. pretiosum podevariarsubstancialmente entre espécies de hospedeiros diferentes (SÁ; PARRA, 1994), o que sugere que os parasitoides são atraídos porcertosinsetosou plantas, ou quealgunshospedeiros são mais adequados para o desenvolvimento dos descendentes (Roriz et al., 2005). Outro fator seria queomaior parasitismoemum determinadohospedeiro pode ser explicado pela experiência do adulto parental nohospedeironatal, associadaà aprendizagem de pistas químicas e visuais, o que, segundo COBERT (1985), pode ocorrer quando o inseto é criado por várias gerações no mesmo hospedeiro (GOULART et al., 2008).

A aceitação pelohospedeironão depende somente de herança genética, mas também de características herdadas ao longo das gerações (PAK et al., 1986; GOUlart et al., 2008). Tais afirmações podem explicar o maior número de ovos parasitados quando se utilizou como hospedeiro A. kuehniella.

A porcentagem de emergência não foi influenciada pelas diferentes cores de cartelas de criação quando se utilizou ovos de $P$. xylostella (brócolis $\mathrm{F}_{6,85}=0,81 ; \mathrm{P}=0,5667$ ) (couve $\mathrm{F}_{6,86}=1,56 ; \mathrm{P}=0,1697$ ) (Tabela 3). Quando foram utilizados ovos de $A$. kuehniella $\left(\mathrm{F}_{6,101}=6,72 ; \mathrm{P}<0,0001\right)$ ocorreu diferença significativa entre os tratamentos e a cor rosa foi a que proporcionou menor número de insetos emergidos, $(70 \%)$ (Tabela 3). Resultados superiores para porcentagem de emergência de T. pretiosum em ovos de A. kuehniella ocorreram quando foram utilizadas cartelas de coloração azul, verde, branco e vermelho, obtendo 97,3; 99,6; 99,1 e 96,6\% de porcentagem de emergência, valores estes que se aproximam das cartelas de cores amarela $(94,1 \%)$ e preta $(80,5)$ (Tabela 3$)$.

Tabela 1 - Análise de variância do efeito dos hospedeiros e de cores de cartelas no parasitismo de Trichogramma pretiosum.

\begin{tabular}{lccccc}
\hline Fonte de variação & GL & SQ & QM & F & P \\
\hline Hospedeiros & 2 & 98,88 & 98,88 & 118,41 & $<0,0001^{*}$ \\
Cartelas & 6 & 12,87 & 2,14 & 2,57 & $0,0207^{*}$ \\
Hosp. $x$ cart. & 12 & 15,27 & 2,54 & 3,05 & $0,0073^{*}$ \\
Erro & 252 & 149,47 & 0,83 & & \\
\hline
\end{tabular}

GL, grau de liberdade; SQ, soma dos quadrados; QM, quadrado médio.

*significativo $(\mathrm{P}<0,05)$.

Tabela 2 - Número de ovos parasitados de Anagasta kuehniella e Plutella xylostella criada em couve e brócolis por Trichogramma pretiosum em diferentes cores de cartelas.

\begin{tabular}{lccc}
\hline \multirow{2}{*}{ Cores de cartelas } & \multicolumn{3}{c}{ Hospedeiros } \\
\cline { 2 - 4 } & Anagasta kuehniella & Plutella xylostella couve & Plutella xylostella brócolis \\
\hline Azul & $22,1 \pm 1,54 \mathrm{Aa}$ & $9,1 \pm 1,69 \mathrm{Ba}$ & $5,2 \pm 1,00 \mathrm{Ba}$ \\
Verde & $21,0 \pm 3,69 \mathrm{Aa}$ & $5,8 \pm 1,16 \mathrm{Ba}$ & $6,3 \pm 0,99 \mathrm{Ba}$ \\
Branco & $20,1 \pm 2,22 \mathrm{Aa}$ & $6,3 \pm 1,02 \mathrm{Ba}$ & $3,2 \pm 0,90 \mathrm{Ba}$ \\
Rosa & $19,9 \pm 1,88 \mathrm{Aa}$ & $9,6 \pm 1,61 \mathrm{Ba}$ & $8,4 \pm 1,10 \mathrm{Ba}$ \\
Vermelho & $19,8 \pm 2,84 \mathrm{Aab}$ & $9,4 \pm 1,33 \mathrm{Ba}$ & $7,2 \pm 1,00 \mathrm{Ba}$ \\
Amarelo & $13,9 \pm 1,41 \mathrm{Aab}$ & $8,1 \pm 0,91 \mathrm{Ba}$ & $7,1 \pm 0,95 \mathrm{Ba}$ \\
Preto & $9,9 \pm 2,09 \mathrm{Ab}$ & $8,6 \pm 1,87 \mathrm{Aa}$ & $6,7 \pm 1,87 \mathrm{Aa}$ \\
\hline
\end{tabular}

${ }^{1}$ Médias \pm erro padrão seguidas pela mesma letra minúscula na coluna e maiúscula na linha não diferem pelo teste de Tukey $(\mathrm{P}<0,05)$. 
Tabela 3 - Análise de variância do efeito dos hospedeiros e de cores de cartelas na porcentagem de emergência de Trichogramma pretiosum.

\begin{tabular}{lccccc}
\hline Fonte de variação & GL & SQ & QM & F & P \\
\hline Hospedeiros & 2 & 16,99 & 8,49 & 5,53 & $0,0044^{*}$ \\
Cartelas & 6 & 32,87 & 5,47 & 3,57 & $0,0021^{*}$ \\
Hosp. $x$ cart. & 12 & 39,49 & 3,31 & 2,16 & $0,0141^{*}$ \\
Erro & 252 & 386,92 & 1,53 & & \\
\hline
\end{tabular}

GL, grau de liberdade; SQ, soma dos quadrados; QM, quadrado médio.

*significativo $(\mathrm{P}<0,05)$.

Tabela 4 - Porcentagem de emergência de Trichogramma pretiosum submetido ao parasitismo em ovos de Anagasta kuehniella e Plutella xylostella sob diferentes cores de cartelas.

\begin{tabular}{|c|c|c|c|}
\hline \multirow{2}{*}{ Cores de cartelas } & \multicolumn{3}{|l|}{ Hospedeiros } \\
\hline & Anagasta kuehniella & Plutella xylostella couve & Plutella xylostella brócolis \\
\hline Azul & $97,3 \pm 1,95 \mathrm{Aa}$ & $80,3 \pm 8,09 \mathrm{ABa}$ & $69,1 \pm 8,46 \mathrm{Ba}$ \\
\hline Verde & $99,6 \pm 0,38 \mathrm{Aa}$ & $94,8 \pm 3,79$ Аа & $87,0 \pm 7,26 \mathrm{Aa}$ \\
\hline Branco & $99,1 \pm 0,70 \mathrm{Aa}$ & $91,3 \pm 3,94 \mathrm{ABa}$ & $75,7 \pm 12,15 \mathrm{Ba}$ \\
\hline Rosa & $70,0 \pm 6,60 \mathrm{Bb}$ & $89,2 \pm 7,15$ Аа & $77,9 \pm 5,33 \mathrm{ABa}$ \\
\hline Vermelho & $96,6 \pm 3,36 \mathrm{Aa}$ & $96,9 \pm 1,25 \mathrm{ABa}$ & $84,5 \pm 4,94 \mathrm{Ba}$ \\
\hline Amarelo & $94,1 \pm 3,87 \mathrm{Aab}$ & $97,6 \pm 1,46 \mathrm{Aa}$ & $83,2 \pm 7,93 \mathrm{Aa}$ \\
\hline Preto & $80,5 \pm 8,66 \mathrm{Aab}$ & $93,9 \pm 3,01 \mathrm{Aa}$ & $95,5 \pm 3,53 \mathrm{Aa}$ \\
\hline
\end{tabular}

${ }^{1}$ Médias \pm erro padrão seguidas pela mesma letra minúscula na coluna e maiúscula na linha não diferem pelo teste de Tukey $(\mathrm{P}<0,05)$.

No controle de qualidade de produção massal de Trichogramma spp., a viabilidadeéconsiderada satisfatória quando o percentual de adultos emergidos for superior a 85\% (AlmEIDA, 1998). Fato esse só não observado neste trabalho nas cartelas de coloração rosa, quando se utilizou como hospedeiros ovos de A. kuehniella.

Há influência das cores branca, laranja, amarela, vermelha e verde em cartelas na criação de $T$. chilonis Ishii, 1941 (Hymenoptera: Trichogrammatidae), e as cores verde e a branca são as mais adequadas para a criação massal deste parasitoide (BAITHA; SINHA, 2002).

Apesar das várias cores utilizadas no trabalho, os adultos de T. pretiosum apresentaram comportamento de parasitismo semelhante tanto em ovos de A. kuehniella como de P. xylostella, porém, testes de preferência devem ser realizados para se determinar quais cores são as preferidas pelo parasitoide, para então confirmar a eficiência destas em uma futura criação massal em laboratório.

\section{CONCLUSÕES}

A linhagem Tp-8 de T. pretiosum apresenta melhor adaptação em ovos de A. kuehniella.

Os substratos de alimentação de $P$. xylostella (couve e brócolis) não influenciam o parasitismo de T. pretiosum.

As cores de cartelas indicadas para a condução da criação massal de T. pretiosum são azul, verde e branco.

\section{REFERÊNCIAS}

ALMEIDA, R.P. Controle de qualidade na produção de Trichogramma. In: ALMEIDA, R.P.; SILVA, C.A.D.; MEDEIROS, M.B. (Ed.) Biotecnologia de produção massal e manejo de Trichogramma para o controle biológico de pragas. Campina Grande: EMBRAPA-CNPA,1998. p.26-34.

AQUINO, M.F.S. Estudo do comportamento de busca e seleção de hospedeiros dos parasitoides de ovos Trissolcus basalis e Telenomus podisi (Hymenoptera: Scelionidae). 2011. 100f. Dissertação (Mestrado em Biologia Animal) - Universidade de Brasília, Brasília, 2011.

BERNAYS, E.A.; CHAPMAN, R.F. Host-plant selection by phytophagous insects. New York: Chapman \& Hall, 1994. $312 \mathrm{p}$.

BAITHA, A.; SINHA, O.K. Response of Trichogramma chilonis Ishii to different colours of trichocards. Indian Journal of Sugarcane Technology, v.17, n.1/2, p.64-67, 2002.

COBERT, S.A. Insect chemosensory responses: a chemical legacy hypothesis. Ecological Entomology, v.10, p.143153, 1985.

FLANDERS, S.E. Biological control of the codling moth (Carpocapsa pomonella). Journal of Economic Entomology, v.20, n.1, p.644, 1927.

FUENTES, S.F. Produccion y uso de Trichogramma como regulador de plagas. Lima: RAAA, 1994. 192p.

GOULART, R.M.; DE BORTOLI, S.A.; THULER, R.T.; PRATISSOLI, D.; VIANA, C.L.T.P.; VOLPE, H.X.L. Avaliação da seletividade de inseticidas a Trichogramma spp. 
(Hymenoptera: Trichogrammatidade) em diferentes hospedeiros. Arquivos do Instituto Biológico, São Paulo, v.75, n.1, p.69-77, 2008.

GOULART, R.M. Criação de Trichogramma spp. In: DE BORTOLI, S.A. (Ed.). Criação de insetos: da base à biofábrica. Jaboticabal: FCAV, 2009. Parte 2, p.127-134.

GOULART, R.M.; VOLPE, H.X.L.; VACARI, A.M.; THULER, R.T.; DE BORTOLI, S.A. Insecticide selectivity to two species of Trichogramma in three different hosts, as determined by IOBC/WPRS methodology. Pest Management Science, v.68, n.2, p.240-244, 2011.

HAJI, F.N.P.; FREIRE, L.C.L.; ROA, F.G.; SILVA, C.N.; SOUZA JÚNIOR, M.M.; SILVA, M.I.V. Manejo integrado de Scrobipalpuloides absoluta (Povolny) (Lepidoptera: Gelechiidae) no Submédio São Francisco. Anais da Sociedade Entomológica do Brasil, v.24, n.3, p.587-591, 1995.

HOLLINGSWORTH, J.P.; HARTSTACK JR, A.W.; LINGREN, P.D. The spectral response of Campoletis perdistinctus. Journal of Economic Entomology, v.63, n.6, p.1758-1761, 1970.

LUNAU, K. Color saturation triggers innate reactions to flower signals: flower-dummy experiments with bumblebees. Journal of Comparative Phisiology, v.166, n.6, p.27-834, 1990.

PAK, G.A.; BUIS, H.C.E.M.; HECK, I.C.C.; HERMANS, M. L. G.. Behavioural variations among strains of Trichogramma spp.: hostage selection. Entomologia Experimentalis et Applicata, v.40, n.3, p. 247-258, 1986.

PARRA, J.R.P. Técnicas de criação de Anagasta kuehniella, hospedeiro alternativo para produção de Trichogramma. In: PARRA, J.R.P.; ZUCCHI, R.A. (Ed.). Trichogramma e controle biológico aplicado. Piracicaba: FEALQ, 1997. p.121-150.

PARRA, J.R.P.; BOTELHO, M.P.S.M.; CORRÊA-FEREREIRA, B.S.; BENTO, J.M. Controle biológico no Brasil: predadores e parasitoides. São Paulo: Manole, 2002. $635 p$.

PEREIRA, F.F.; BARROS, R.; PRATISSOLI, D.; PARRA, J.R.P. Biologia e exigências térmicas de Trichogramma pretiosum Riley e T. exiguum Pinto \& Platner (Hymenoptera: Trichogrammatidae) criados em ovos de Plutella xylostella (L.) (Lepidoptera: Plutellidae). Neotropical Entomology, v.33, n.2, p.231-236, 2004.

PINTO, J.D. Systematics of the North American species of Trichogramma Westwood (Hymenoptera:

Trichogrammatidae). Washington: Entomological Society of Washington, 1999. v.22, p.1-287.

POLANCZYK, R.A.; PRATISSOLI, D.; HOLTZ, A.M.; PEREIRA, C.L.T.; FURTADO, I.S.A. Efeito da idade de Trichogramma exiguum e do desenvolvimento embrionário da traça-das-crucíferas sobre as características biológicas do parasitoide. Acta Scientiarum. Biological Sciences, v.29, n.2, p.161-166, 2007.

PRATISSOLI, D.; PEREIRA, F.F.; BARROS, R.; PARRA, J.R.P.; PEREIRA, C.L.T. Parasitismo de Trichogramma pretiosum em ovos da traça-das-crucíferas sob diferentes temperaturas. Horticultura Brasileira, v.22, n.4, p.754-757, 2004.

QUERINO, R.B.; ZUCCHI, R.A. New species of Trichogramma Westwood (Hymenoptera:

Trichogrammatidae) associated with lepidopterous eggs in Brazil. Zootaxa, v.163, p.1-10, 2003.

ROMEIS, J.; SHANOVER, T.G.; ZEBITZ, C.P.W Response of Trichogramma egg parasitoids to colored sticky traps. Biocontrol, v.43, p.17-27, 1998.

RORIZ, V.; OLIVEIRA, L.; GARCIA, P. Host suitability and preference of Trichogramma cordubensis (Hymenoptera: Trichogrammatidae). Biological Control, v.36, p.331-336, 2005.

SÁ, L.A.N.; PARRA, J.R.P. Natural parasitism of Spodoptera frugiperda and Helicoverpa zea (Lepidoptera: Noctuidae) eggs in corn by Trichogramma pretiosum (Hymenoptera: Trichogrammatidae) in Brazil. Florida Entomologist, v.77, p.185-188, 1994.

SAS INSTITUTE. SAS/STAT User`s Guide, version 9.00 TS level 2MO. Cary, NC: SAS Institute Inc., 2002.

SEGURA, D.F.; VISCARRET, M.M.; PALADINO, L.Z.C.; OVRUSKI, S.M.; CLADERA, J.L. Animal Behaviour, v.74, n.1, p.131-142, 2007.

THULER, R.T. Criação de Plutella xylostella. In: DE BORTOLI, S.A. (Ed.). Criação de insetos: da base à biofábrica. Jaboticabal: FCAV, 2009. Parte 2, p.58-68.

THULER, R.T.; VOLPE, H.X.L.; DE BORTOLI, S.A.; GOULART, R.M.; VIANA, C.L.T.P. Metodologia para avaliação da preferência hospedeira de parasitoides do gênero Trichogramma Westwood. Boletin de Sanidad Vegetal Plagas, v.33, n.3, p.333-340, 2007.

THULER, R.T.; DE BORTOLI, S.A.; GOULART, R.M.; VIANA, C.L.T.P.; PRATISSOLI, D. Interação tritrófica e influência de produtos químicos e vegetais no complexo: brássicas $x$ traça-das-crucíferas $x$ parasitoides de ovos. Ciência e Agrotecnologia, v.32, n.4, p.1154-1160, 2008.

VINSON, S.B. The behavior of parasitoids. In: KERTUT, G.A.; GILBERT, L.I. (Ed.). Comprehensive insect physiology, biochemistry and pharmacology. New York: Pergamon Press, 1985. p.417-469.

VOLPE, H.X.L.; DE BORTOLI, S.A.; THULER, R.T.; VIANA, C.L.T.P.; GOULART, R.M. Avaliação de características biológicas de Trichogramma pretiosum Riley (Hymenoptera: Trichogrammatidae) criado em três 
hospedeiros. Arquivos do Instituto Biológico, São Paulo, v.73, n.3, p.311-315, 2006.

ZUCCHI, R.A.; MONTEIRO, R.C. O gênero Trichogramma na América do Sul. In: PARRA, J.R.P.; ZUCCHI, R.A.
(Ed.). Trichogramma e o controle biológico aplicado. Piracicaba: FEALQ, 1997. p.41-66.

Recebido em 21/9/10

Aceito em 30/11/11 